Maria Paula Cerqueira Gomes 1,2 Maria Cristina Ventura Couto 2,3 Vera Lúcia Edais Pepe 4

Liz Maria de Almeida 5

Pedro Gabriel Godinho Delgado 1,6 Evandro da Silva Freire Coutinho 7

\section{Censo dos pacientes internados em uma instituição asilar no Estado do Rio de Janeiro: dados preliminares}

\author{
Census of hospitalized patients in \\ a psychiatric institution of Rio de Janeiro State: \\ preliminary data
}

1 Departamento de Psiquiatria e Medicina Legal, Faculdade de Medicina, Universidade Federal do Rio de Janeiro. Rua Venceslau Brás 71, Rio de Janeiro, $R J$ 22290-140, Brasil.

2 Assessoria de Saúde Mental, Secretaria de Estado de Saúde do Rio de Janeiro. Rua México 128, Rio de Janeiro, $R J$ 20031-142, Brasil.

3 Instituto de Psiquiatria, Universidade Federal do Rio de Janeiro. Rua Venceslau Brás 71, Rio de Janeiro, RJ 22290-140, Brasil.

4 Departamento de Administração e Planejamento em Saúde, Escola Nacional de Saúde Pública, Fundação Oswaldo Cruz. Av. Leopoldo Bulhões 1480, Rio de Janeiro, RJ 21041-210, Brasil.

5 Núcleo de Estudos de Saúde Coletiva, Centro de Ciências da Saúde, Universidade Federal do Rio de Janeiro. C. P. 68037, Rio de Janeiro, $R J$ 21941-590, Brasil. 6 Secretaria de Assistência à Saúde, Ministério da Saúde. Esplanada dos Ministérios, Bloco E, Brasília, DF 20031-142, Brasil.

7 Departamento de Epidemiologia e Métodos Quantitativos em Saúde Escola Nacional de Saúde Pública, Fundação Oswaldo Cruz. Av. Leopoldo Bulhões 1480, Rio de Janeiro, RJ 21041-210, Brasil.

\begin{abstract}
Rio de Janeiro State has one of the largest networks of psychiatric hospitals in Brazil, one of them the Dr. Eiras Paracambi Hospital (CSDE-P). In late 2000, the current Mental Health Advisory Division of the Rio de Janeiro State Health Department conducted a clinical and psychosocial census of patients hospitalized in the CSDE-P. The hospital population consisted of 1,494 individuals, the majority men (53.4\%), single (81.6\%), working-age (66.4\%), and with limited schooling (only $11.5 \%$ with a complete primary education or more). Most had been in hospital for at least two years (77.6\%), received visits from relatives (60.5\%), but did not have hospital leave (73.8\%). The predominant diagnoses were schizophrenia (53.6\%) and mental retardation (26.4\%). The main therapeutic approach was psychiatric (84.1\%). Only 13.3\% took part in systematic activities during the day, although $36 \%$ were in a condition to do so. CSDE-P is a megahospital where most patients have precarious social links, demanding treatment strategies which take this characteristic into account.
\end{abstract}

Key words Mentally Ill Persons; Mental Health; Inpatients

Resumo O Estado do Rio de Janeiro concentra uma das maiores redes de hospitais psiquiátricos do país, sendo um deles a Casa de Saúde Dr. Eiras/Paracambi (CSDE-P). A atual Assessoria de Saúde Mental da Secretaria de Estado de Saude do Estado do Rio de Janeiro, realizou em fins de 2000, o Censo Clínico e Psicossocial dos Pacientes Internados na CSDE-P. Encontrou uma população de 1494 indivíduos, com predomínio de homens (53,4\%), solteiros (81,6\%), faixa etária produtiva (66,4\%) e baixa escolaridade (11,5\% com primeiro grau completo ou mais). A maioria tinha um tempo de internação superior/igual a 2 anos (77,6\%), recebiam visitas de familiares (60,5\%) mas não saíam de licença $(73,8 \%)$. Os diagnósticos predominantes foram as esquizofrenias (53,6\%) e o retardo mental (26,4\%). A principal abordagem terapêutica foi a psiquiátrica (84,1\%). Realizavam atividades sistemáticas durante o dia 13,3\% dos pacientes, embora cerca de 36\% apresentassem bons indicativos de condições de autonomia. A CSDE-P é um macro hospital, onde a maioria dos pacientes encontra-se desterritorializada e com laços sociais precários, exigindo a construção de estratégias de cuidados que levem em conta essa particularidade.

Palavras-chave Pessoas Mentalmente Doentes; Saúde Mental; Pacientes Internados 


\section{Introdução}

A partir da década de 90, observa-se um processo de reorientação do modelo assistencial no campo da saúde mental. Nesse contexto, as coordenações de saúde mental, estaduais e municipais no país, estão envolvidas, ainda que em graus diferentes, com a criação de condições técnicas e políticas que garantam o direito ao tratamento e à organização de uma rede de atenção à saúde integral de base comunitária, através de dispositivos sanitários e socioculturais da vida do indivíduo (Delgado et al., 2001).

Nesse contexto de reforma do modelo assistencial em saúde mental são necessários indicadores clínicos, epidemiológicos, sociais e de organização de serviços. Além disso, é fundamental a criação e implantação de mecanismos de controle e avaliação da rede hospitalar pública e contratada pelo Sistema Único de Saúde (SUS) (Delgado et al., 2001).

O Estado do Rio de Janeiro concentra uma das maiores redes de hospitais psiquiátricos de grande porte no país, consumindo a maior parcela de recursos destinados a essa área. O Município de Paracambi, Rio de Janeiro, localizado na região metropolitana do Estado do Rio de Janeiro, contava no ano de 2000, com 1.670 leitos psiquiátricos contratados pelo SUS em dois hospitais. Um deles é a Casa de Saúde Dr. Eiras - Paracambi (CSDE-P) com 1.510 leitos. O município responde pelo maior número de $\mathrm{Au}$ torizações de Internação Hospitalar (AIH) faturadas, tendo recebido no ano de 1999, cerca de $17 \%$ de todos os recursos de saúde mental destinados a internações hospitalares do estado. Apesar do grande volume de pacientes internados e recursos recebidos, o resultado da assistência prestada vem sendo questionada por sucessivos gestores estaduais, federais e órgãos não-governamentais (Comissão de Direitos Humanos, 2000; IFB, 2000).

O Plano Emergencial para Reorientação da Assistência na CSDE-P (Rio de Janeiro, 2000), proposto pela Assessoria de Saúde Mental da Secretaria de Estado de Saúde do Rio de Janeiro (SES-RJ), tem como eixo principal de suas ações a reversão do modelo centrado nas internações psiquiátricas, propondo a oferta de dispositivos extra-hospitalares de base territorial, como referência principal para o tratamento de pacientes portadores de transtornos mentais severos e persistentes. Uma da primeiras ações desse Plano foi a realização do Censo Clínico e Psicossocial dos Pacientes Internados na $C S D E-P$, nos meses de novembro e dezembro de 2000. Este Censo teve por objetivo produzir informações técnicas para auxiliar a reorienta- ção do modelo assistencial existente na CSDE$\mathrm{P}$ e em seu entorno, formada por municípios populosos e desprovidos de rede pública eficaz. Além disso, o censo buscou fornecer um diagnóstico clínico, psicossocial e socioeconômico com vistas a estabelecer um perfil da clientela internada e subsidiar a construção de projetos terapêuticos individualizados.

Neste artigo, apresentaremos os principais resultados obtidos numa análise preliminar deste censo.

\section{Material e métodos}

O instrumento de pesquisa e seu respectivo manual, tomaram como base o instrumento e manual utilizados para a execução do censo da população de internos nos hospitais psiquiátricos da cidade do Rio de Janeiro (SMS-RJ, 1997). Foram acrescidos campos referentes à avaliação clínica, psiquiátrica e indicativos da autonomia do paciente.

Oitenta e quatro profissionais de saúde mental foram selecionados e treinados para a execução do censo, sendo que $50 \%$ da equipe era composta por médicos psiquiatras. Foram formadas 7 equipes de trabalho, cada uma com dois coordenadores de campo e dez pesquisadores. Os dados coletados tiveram como fontes entrevistas e exames com o paciente, entrevistas com profissionais de saúde da CSDE-P e o prontuário médico. Os dados foram armazenados em banco de dados e analisados utilizando-se o programa Epi Info (versão 6.04 - CDC/ WHO, 1996). Nessa análise preliminar foram estimadas proporções e médias das variáveis mais importantes, estratificadas por sexo.

\section{Resultados}

O censo obteve informações sobre 1494 pacientes, sendo 696 mulheres $(46,6 \%)$ e 798 homens $(53,4 \%)$. Na Tabela 1, são apresentadas algumas características gerais da população de pacientes, por sexo. Cerca de dois terços dos pacientes tinham menos de 50 anos, sendo a população feminina mais velha. Quanto à escolaridade, metade da população internada era analfabeta. Havia oito homens e apenas uma mulher com 3o grau completo.

Dentre os pacientes, $42 \%$ perderam seus vínculos referenciais de moradia, sendo considerados moradores do hospital. Entre aqueles que ainda tinham vínculo referencial de moradia, predominavam os oriundos dos municípios do Rio de Janeiro (23,9\%) e de Nova Iguaçu $(21,9 \%)$. 
Características sócio-demográficas dos pacientes da Casa de Saúde Dr. Eiras.

Paracambi, Rio de Janeiro, Brasil, por sexo.

\begin{tabular}{|c|c|c|c|c|c|c|}
\hline \multirow[t]{2}{*}{ Variável } & \multicolumn{2}{|c|}{ Masculino } & \multicolumn{2}{|c|}{ Feminino } & \multicolumn{2}{|c|}{ Total } \\
\hline & $\mathrm{n}$ & $\%$ & $\mathrm{n}$ & $\%$ & $n$ & $\%$ \\
\hline \multicolumn{7}{|l|}{ Idade (anos) } \\
\hline $18-29$ & 92 & 11,6 & 65 & 9,4 & 157 & 10,5 \\
\hline $30-39$ & 205 & 25,8 & 168 & 24,2 & 373 & 25,0 \\
\hline $40-49$ & 263 & 33,0 & 197 & 28,4 & 460 & 30,9 \\
\hline $50-59$ & 144 & 18,1 & 149 & 21,5 & 293 & 19,7 \\
\hline 60 e mais & 92 & 11,6 & 115 & 16,6 & 207 & 13,9 \\
\hline \multicolumn{7}{|l|}{ Escolaridade } \\
\hline Analfabeto & 358 & 49,6 & 299 & 47,1 & 657 & 48,4 \\
\hline Lê e escreve & 143 & 19,8 & 102 & 16,1 & 245 & 18,1 \\
\hline 1ㅇ grau incompleto & 142 & 19,7 & 158 & 24,9 & 300 & 22,1 \\
\hline 1ㅇ grau completo & 55 & 7,6 & 64 & 10,1 & 119 & 8,8 \\
\hline 2o grau completo e + & 24 & 3,3 & 12 & 1,8 & 36 & 2,7 \\
\hline \multicolumn{7}{|l|}{ Situação conjugal } \\
\hline Solteiro & 688 & 88,6 & 496 & 73,6 & 1.184 & 81,6 \\
\hline Casado ou união & 48 & 6,2 & 85 & 12,6 & 133 & 9,2 \\
\hline Separado ou divorciado & 33 & 4,2 & 54 & 8,0 & 87 & 6,0 \\
\hline Viúvo & 8 & 1,0 & 39 & 5,8 & 47 & 3,2 \\
\hline \multicolumn{7}{|l|}{ Moradia } \\
\hline Fora do hospital & 122 & 15,4 & 137 & 19,7 & 259 & 17,4 \\
\hline No hospital, com casa fora & 342 & 43,3 & 214 & 30,8 & 556 & 37,5 \\
\hline No hospital, sem casa fora & 303 & 38,4 & 330 & 47,6 & 633 & 42,7 \\
\hline Rua e albergue & 23 & 2,9 & 13 & 1,9 & 36 & 2,4 \\
\hline \multicolumn{7}{|l|}{ Tempo de internação } \\
\hline até 1 mês & 9 & 1,1 & 9 & 1,3 & 18 & 1,2 \\
\hline $1-6$ meses & 75 & 9,4 & 78 & 11,2 & 153 & 10,2 \\
\hline 6-12 meses & 38 & 4,8 & 31 & 4,5 & 69 & 4,6 \\
\hline $1-2$ anos & 48 & 6,0 & 45 & 6,5 & 93 & 6,2 \\
\hline 2-5 anos & 154 & 19,3 & 190 & 27,3 & 344 & 23,0 \\
\hline $5-10$ anos & 129 & 16,2 & 142 & 20,4 & 271 & 18,1 \\
\hline mais de 10 anos & 345 & 43,2 & 201 & 28,9 & 546 & 36,5 \\
\hline \multicolumn{7}{|l|}{ Renda } \\
\hline Tem & 354 & 48,8 & 255 & 38,7 & 609 & 44,0 \\
\hline Não tem & 371 & 51,2 & 404 & 61,3 & 775 & 56,0 \\
\hline \multicolumn{7}{|l|}{ Licença } \\
\hline Sai & 218 & 27,4 & 171 & 24,7 & 389 & 26,2 \\
\hline Não sai & 577 & 72,6 & 521 & 75,3 & 1.098 & 73,8 \\
\hline \multicolumn{7}{|l|}{ Visita de familiares } \\
\hline Recebe & 529 & 66,3 & 374 & 53,9 & 903 & 60,5 \\
\hline Não recebe & 269 & 33,7 & 320 & 46,1 & 589 & 39,5 \\
\hline
\end{tabular}

Fonte: SES-RJ/MS (2001). 
Observou-se que uma proporção maior de homens não estabeleceu uma relação afetiva estável ao longo da vida (solteiros), quando comparados com as mulheres $(88,6 \%$ vs $73,6 \%)$. No momento do censo apenas $6,2 \%$ dos homens e $12,6 \%$ das mulheres estavam casados ou unidos consensualmente.

Oitenta por cento dos pacientes residiam no próprio hospital. O critério para considerar um paciente morador do hospital foi um tempo de permanência hospitalar e ininterrupto maior que dois anos na CSDE-P, ou que foram transferidos diretamente de outra instituição psiquiátrica e também somavam mais de 24 meses de internação. Quarenta e dois por cento desses pacientes internados não tinham lugar para ficar fora do hospital.

Mais de três quartos dos pacientes estavam há mais de dois anos no hospital, e um terço há mais de dez anos. Deve-se assinalar a maior proporção de homens do que mulheres internados há mais de dez anos $(43,2 \%$ vs $28,9 \%$, respectivamente).

Quarenta e quatro por cento dos pacientes tinham alguma fonte de renda, sendo que $63 \%$ deles tinham a previdência como fonte desta renda. Existia uma proporção maior de mulheres sem qualquer fonte de renda.

Cerca de $74 \%$ dos pacientes internados não saíam de licença, não havendo diferença por sexo. Sessenta por cento dos pacientes recebiam visitas de familiares, sendo que entre esses, $17,3 \%$ recebiam visitas semanalmente e $32,8 \%$ mensalmente.

O diagnóstico principal mais freqüentemente encontrado nos prontuários foi a esquizofrenia $(53,6 \%)$, seguido do retardo mental $(26,4 \%)$. $\mathrm{O}$ alcoolismo foi referido para apenas $1,8 \%$ dos pacientes.

Apenas 2,3\% dos pacientes não faziam uso de medicação, enquanto $87 \%$ recebiam prescrição múltipla. A média de medicamentos prescritos por paciente foi de 3,3. Dentre os medicamentos prescritos, $40 \%$ eram antipsicóticos e $21 \%$ antiepilépticos. Medicamentos usados para controlar os efeitos adversos dos antipsicóticos somaram $17 \%$ das prescrições, enquanto os ansiolíticos representaram $12 \%$. Com relação aos efeitos adversos da medicação, observou-se $12 \%$ de discinesia tardia, $5 \%$ com acatisia e $11 \%$ com síndrome extra-piramidal.

A equipe médica do censo identificou $7 \%$ dos pacientes com estado geral debilitado, $9 \%$ com sinais evidentes de emagrecimento, $37 \%$ com alterações de pele e fâneros e $68 \%$ com a arcada dentária em péssimo estado.

Os dados obtidos revelaram a predominância da abordagem psiquiátrica em caráter re- gular e semanal em $84,1 \%$ dos casos e do clínico geral em $12 \%$. Quanto às demais abordagens - psicologia, fisioterapia, serviço social e terapia ocupacional, todos os percentuais foram inferiores a $10 \%$. Apenas $13,3 \%$ dos pacientes realizavam atividades sistemáticas durante o dia.

Cerca de $36 \%$ dos pacientes foram considerados como tendo bons indicativos de condições de autonomia, isto é, respondiam adequadamente quando interpelados, andavam e alimentavam-se sozinhos, cuidavam da higiene pessoal e mostravam aparência e vestimentas adequadas.

\section{Discussão}

A população estudada é composta, em sua maioria, por indivíduos solteiros, oriundos do Estado do Rio de Janeiro, na faixa etária produtiva e com baixo grau de escolaridade. A maior parte deles tem um tempo de internação superior a dois anos, sendo que um terço está internado há mais de uma década. Embora recebam visitas de familiares, esses pacientes em geral não saem de licença. Quase a metade desses pacientes tem alguma forma de renda.

Um censo realizado em 1989 numa instituição asilar no Rio de Janeiro - Colônia Juliano Moreira (CJM) (Keusen, 1995; Keusen \& Lima, 1994), encontrou uma população mais velha, com menor grau de escolaridade, mas com um perfil conjugal semelhante ao encontrado na CSDE-P. Na CJM o tempo médio de internação foi superior ao observado em nosso estudo, assim como a proporção de pacientes que não recebiam visitas.

Um censo realizado nos hospitais psiquiátricos do Município do Rio de Janeiro (Silva et al., 1999), encontrou uma população mais jovem e com menor proporção de solteiros, maior nível de escolaridade e maior proporção com algum tipo de renda do que o observado na CSDE-P.

A CSDE-P e a CJM apresentam um perfil diagnóstico semelhante, porém diverso daquele encontrado no CHRJ. Neste último havia menos pacientes oligofrênicos e mais distúrbios associados ao uso de álcool.

A CSDE-P é um macro hospital, cuja ocupação de leitos demonstra pouca dinamicidade $\mathrm{e}$ resolutividade exigidas nos instrumentos normativos no campo da saúde mental. O conjunto desses pacientes encontra-se, em sua maioria, desterritorializado e com laços sociais precários ou inexistentes, exigindo a construção de estratégias de cuidados que levem em conta essa particularidade. 


\section{Agradecimentos}

Os autores agradecem à toda equipe de pesquisadores e de suporte ao Censo, à Secretaria de Estado de Saúde, à Coordenação de Saúde Mental do Ministério da Saúde, bem como aos pacientes da Casa de Saúde Dr. Eiras, Paracambi, sem os quais este Censo não teria se realizado.

\section{Referências}

CDC (Centers for Disease Control and Prevention)/ WHO (World Health Organization), 1996. Epi Info 6, Version 6.04. A Word Processing, Database, and Statistics Program for Public Health. Atlanta: CDC/ Geneva: WHO.

COMISSÃO DE DIREITOS HUMANOS, 2000. Relatório da I Caravana de Cidadania. Brasília: Câmara dos Deputados. (mimeo.)

DELGADO, P. G. G.; GOMES, M. P. C. \& COUTINHO, E. S. F., 2001. Novos rumos nas políticas públicas de saúde mental no Brasil. Cadernos de Saúde Pública, 17:452-453.

IFB (Instituto Franco Basaglia), 2000/2001. Reportagem Dr. Eiras-Paracambi. Boletim Dezoito de Maio, 17:1-12.

KEUSEN, A. L., 1995. Colônia Juliano Moreira: Do Asilo ao Complexo Multiassistencial. Tese de Doutorado, Rio de Janeiro: Instituto de Psiquiatria, Universidade Federal do Rio de Janeiro.

KEUSEN, A. L. \& LIMA L. A., 1989. Perfil da clientela de um asilo: Colônia Juliano Moreira. Jornal Brasileiro de Psiquiatria, 43:281-283.

RIO DE JANEIRO (Estado), 2000. Resolução 1554. Constitui comissão de acompanhamento do processo de alta dos pacientes internados na Casa de Saúde Dr. Eiras, Paracambi. Rio de Janeiro: Diário Oficial do Estado do Rio de Janeiro, ano XXVI, p. 23, 8 nov. pt. 1.
SES-RJ (Secretaria Estadual de Saúde do Rio de Janeiro)/MS (Ministério da Saúde), 2001. Censo Clínico e Psicossocial dos Pacientes da Casa de Saúde Doutor Eiras, Paracambi. Relatório Final. Rio de Janeiro: SES-RJ/MS

SILVA, J. P. L.; COUTINHO, E. S. F. \& AMARANTE, P. D., 1999. Perfil demográfico e socioeconômico da população de internos dos hospitais psiquiátricos da cidade do Rio de Janeiro. Cadernos de Saúde Pública, 15:505-511.

SMS-RJ (Secretaria Municipal de Saúde do Rio de Janeiro), 1995. Censo da População de Internos dos Hospitais Psiquiátricos da Cidade do Rio de Janeiro. Rio de Janeiro: SMS-RJ. (mimeo.)

Recebido em 1 de outubro de 2001

Versão final reapresentada em 8 de fevereiro de 2002

Aprovado em 22 de maio de 2002 